\title{
A NOTE ON LINEAR HOMOGENEOUS DIOPHANTINE EQUATIONS
}

\author{
L. W. GRIFFITHS
}

In this paper the coefficients $a_{i j}$ in the equations

$$
a_{i 1} x_{1}+\cdots+a_{i n} x_{n}=0 \quad(i=1, \cdots, m)
$$

are constant rational integers and all letters denote integers. If $m=n-1$ and the rank is $n-1$ then the complete solution in integers is well known. Thus, if $E_{j}$ is the determinant obtained by deleting the $j$ th column from the matrix of the coefficients, and if $e=\left(E_{1}, \cdots, E_{n}\right)$, then the solution is

$$
x_{j}=(-1)^{i} t E_{j} / e \quad(j=1, \cdots, n),
$$

in which $t$ is an arbitrary integer.

E. T. Bell recently conjectured that if $m<n-1$ and if the rank $r$ is $m$ then the solution is similarly obtained from the system formed by (1) and the equations

$$
\xi_{i 1} x_{1}+\cdots+\xi_{i n} x_{n}=0 \quad(i=1, \cdots, n-m-1),
$$

in which the $\xi_{i j}$ are arbitrary integers. In this paper this conjecture is proved by induction. Since this solution is written down directly from (1) and is fully displayed these results are more usable than those in the literature. ${ }^{1}$

If $r=1$ it can be assumed without limitation that $a_{1} \cdots a_{n} \neq 0$, $\left(a_{1}, \cdots, a_{n}\right)=1$, and at least one of $x_{1}, \cdots, x_{n}$ is not zero. If $n=3$ there are integers $t, y_{1}, y_{2}, y_{3}, d, A_{1}, A_{2}, k_{1}, k_{2}$ such that
(4) $x_{1}=t y_{1}$,
$x_{2}=t y_{2}$,
$x_{3}=t y_{3}$,
$\left(y_{1}, y_{2}, y_{3}\right)=1$,
(5) $a_{1}=d A_{1}$,
$a_{2}=d A_{2}$,
$\left(A_{1}, A_{2}\right)=1$,
$k_{1} A_{2}-k_{2} A_{1}=1$.

Since $\left(d, a_{3}\right)=1$ there is an integer $s$ such that

$$
y_{3}=d s, \quad A_{1} y_{1}+A_{2} y_{2}+a_{3} s=0 .
$$

Then since $\left(A_{1}, A_{2}\right)=1$ there is an integer $r$ such that

$$
y_{1}-a_{3} k_{2} s=A_{2} r, \quad y_{2}+a_{3} k_{1} s=-A_{1} r .
$$

These conditions are also sufficient. Hence the complete solution is

Presented to the Society, April 26, 1946; received by the editors March 18, 1946.

1 Th. Skolem, Diophantische Gleichungen, Ergebnisse der Mathematik und ihrer Grenzgebiete, vol. 5, no. 4, 1938; D. N. Lehmer, Proc. Nat. Acad. Sci. U.S.A. vol. 4 (1919). 
(8)

$$
\begin{aligned}
& x_{1}=t \cdot \frac{1}{d}\left|\begin{array}{cc}
d k_{2} s & -r \\
a_{2} & a_{3}
\end{array}\right|, \\
& x_{2}=-t \cdot \frac{1}{d}\left|\begin{array}{cc}
d k_{1} s & -r \\
a_{1} & a_{3}
\end{array}\right|,
\end{aligned}
$$

$$
x_{3}=t \cdot \frac{1}{d}\left|\begin{array}{cc}
d k_{1} s & d k_{2} s \\
a_{2} & a_{3}
\end{array}\right| \text {. }
$$

Since $\left(d, a_{3}\right)=1$ there are integers $p$ and $P$ such that $-r+p a_{3}=d P$. Hence $d$ is the greatest common divisor of the determinants in (8). Therefore for the particular values $-d k_{1} s,-d k_{2} s, r$ of $\xi_{11}, \xi_{12}, \xi_{13},(8)$ is an instance of (2).

Similarly, if $n=4$ and $d_{1}=\left(a_{1}, a_{2}\right), d_{3}=\left(a_{3}, a_{4}\right)$ then the solution of $a_{1} x_{1}+a_{2} x_{2}+a_{3} x_{3}+a_{4} x_{4}=0$ is equivalent to that of $A_{1} y_{1}+A_{2} y_{2}=-d_{3} S$ and $A_{3} y_{3}+A_{4} y_{4}=d_{1} S$. By the preceding discussion the complete solution for each of these equations is known. The expressions for $S$ are then equated. Thus it is proved that there are integers $k_{1}, \cdots, k_{4}$, $r_{1}, r_{3}, t_{1}, t_{3}, s$ such that the solution is obtained by applying (2) to the matrix

$$
\left[\begin{array}{cccc}
-r_{3} A_{1} & -r_{3} A_{2} & d_{3} k_{3} t_{1} s & d_{3} k_{4} t_{1} s \\
d_{1} k_{1} t_{3} s & d_{1} k_{2} t_{3} s & -r_{1} A_{3} & -r_{1} A_{4} \\
d_{1} A_{1} & d_{1} A_{2} & d_{3} A_{3} & d_{3} A_{4}
\end{array}\right]
$$

If $n \geqq 5$ the notations $x_{j}=t y_{j}(j=1, \cdots, n),\left(y_{1}, \cdots, y_{n}\right)=1$, $a_{j}=\delta \alpha_{j}(j=1, \cdots, n-2), a_{j}=\zeta A_{j}(j=n-1, n),\left(\alpha_{1}, \cdots, \alpha_{n-2}\right)=1$, $\left(A_{n-1}, A_{n}\right)=1, \quad \alpha_{j}=d_{1} A_{j}(j=1, \cdots, n-3),\left(A_{1}, \cdots, A_{n-3}\right)=1$, $\alpha_{n-2}=d_{3} A_{n-3}, \quad \zeta=d_{3} \eta, \quad\left(A_{n-3}, \eta\right)=1$ are used. The solution of $a_{1} x_{1}+\cdots+a_{n} x_{n}=0$ is equivalent to that of $A_{n-1} y_{n-1}+A_{n} y_{n}=\delta S$ and $\alpha_{1} y_{1}+\cdots+\alpha_{n-2} y_{n-2}=-\zeta S$. In particular, if $n=5$ then $y_{4}, y_{5}$, $S$ are obtained as in (8) and $y_{1}, y_{2}, y_{3}, S$ from (9) with $A_{4}$ replaced by $\eta$. If, to simplify the notation, $f_{i j}$ denotes the element in the $i$ th row and $j$ th column of this new matrix then $x_{1}, \cdots, x_{5}$ are obtained by applying (2) to the matrix

$$
\left[\begin{array}{ccccc}
-r_{4} d_{1} A_{1} & -r_{4} d_{1} A_{2} & -r_{4} d_{3} A_{3} & d_{3} \eta q_{4} S_{4} t_{4} & d_{3} \eta q_{5} S_{4} t_{4} \\
\delta f_{11} & \delta f_{12} & \delta f_{13} & f_{14} A_{4} & f_{14} A_{5} \\
\delta f_{21} & \delta f_{22} & \delta f_{23} & f_{24} A_{4} & f_{24} A_{5} \\
\delta d_{1} A_{1} & \delta d_{1} A_{2} & \delta d_{3} A_{3} & d_{3} \eta A_{4} & d_{3} \eta A_{5}
\end{array}\right] .
$$

In general, the matrix for $x_{1}, \cdots, x_{n}$ is obtained from the matrix for $n-1$ variables in precisely the same way as (10) is obtained from (9).

If $1<r<n-1$ it can be assumed without limitation that the first equation satisfies the conditions $a_{1} \cdots a_{n} \neq 0,\left(a_{1}, \cdots, a_{n}\right)=1$, 
of the preceding discussion. If $E_{i}$ denotes $a_{i 1} x_{1}+\cdots+a_{i n} x_{n}$ $(i=1, \cdots, r)$ then a given solution of $E_{1}=0$ is obtained by applying (2) to an appropriate matrix whose $i$ th row is $\alpha_{i 1}, \cdots, \alpha_{i n}$ $(i=1, \cdots, n-2)$ and whose last row is $a_{11}, \cdots, a_{1 n}$. By hypothesis these values of $x_{1}, \cdots, x_{n}$ also satisfy $E_{2}=0$. Therefore the determinant obtained by placing the row $a_{21}, \cdots, a_{2 n}$ under this matrix is zero. Hence, if $A_{i}$ is defined as $\alpha_{i 1} x_{1}+\cdots+\alpha_{i n} x_{n}$ $(i=1, \cdots, n-2)$, then the rank $s$ of the functions $A_{1}, \cdots, A_{n-2}$, $E_{1}, E_{2}$ is less than $n$. Therefore there is a set of $s$ functions from this list on which each remaining function is linearly dependent, with integer coefficients. If indeed the subset includes both $E_{1}$ and $E_{2}$ then the notation can be assigned so that the subset is $A_{1}, \cdots, A_{s-2}, E_{1}$, $E_{2}$. Then there are integers $d, d_{1}, \cdots, d_{s}$ such that $d \neq 0$ and

$$
d A_{n-2}=d_{1} A_{1}+\cdots+d_{s-2} A_{s-2}+d_{s-1} E_{1}+d_{s} E_{2} .
$$

Now the result of applying (2) to the original matrix is the same as the result of applying it to the matrix which is obtained by replacing $\alpha_{n-2, j}$ by $d \alpha_{n-2, j}(j=1, \cdots, n)$. By (11) in this new matrix $d \alpha_{n-2, j}$ may be replaced by the sum $d_{1} \alpha_{1 j}+\cdots+d_{s-2} \alpha_{s-2, j}+d_{s-1} a_{1 j}+d_{s} a_{2 j}$, and hence by $d_{s} a_{2 j}$, and hence by $a_{2 j}$. Again, if the subset includes $E_{2}$ but not $E_{1}$, or if it includes neither $E_{1}$ nor $E_{2}$, or if it includes $E_{1}$ but not $E_{2}$, then in a similar way the matrix can be replaced by a matrix having $a_{11}, \cdots, a_{1 n}$ as last row and $a_{21}, \cdots, a_{2 n}$ as another row. This process can be continued until $a_{i 1}, \cdots, a_{i n}(i=1, \cdots, r)$ appear.

NORTHWESTERN UNIVERSITY 